\section{S11.4 ROLE OF THE GENITAL TRACT MICROBIOME IN SEXUAL AND REPRODUCTIVE HEALTH: REPORT FROM THE KEYSTONE SYMPOSIUM IN CAPE TOWN, 2018}

Janneke van de Wijgert*. University Medical Center Utrecht, Julius Center for Health Sciences and Primary Care, Utrecht, Netherlands

\subsection{6/sextrans-2019-sti.56}

Background The Keystone Symposium on 'Role of the Genital Tract Microbiome in Sexual and Reproductive Health' was held in Cape Town in December 2018.

Methods The aims were to discuss the most current and cutting-edge research findings, consider future directions, stimulate collaborations, and provide a forum for trainees and new investigators to present, learn, and network.

Results/Conclusions All aims were met. The Symposium was particularly successful in bringing researchers in HIV/STI and maternal and child health together. Areas of scientific consensus included: 1) Optimal is a better term to use than normal in the context of vaginal microbiota. L. crispatus-domination is considered optimal. L. iners is sometimes a friend and sometimes a foe. 2) Vaginal microbiota diversity on its own misses important dysbiosis types (such as G. vaginalis-domination). 3) Taxonomic community state types are useful, but we may need to move towards community state types that incorporate pathogenicity (e.g. inflammatory potential), and are therefore more useful from the prevention, diagnosis, treatment and prognosis perspectives. For some key bacterial taxa (e.g. G. vaginalis), we should differentiate between clades. In the meantime, we should use precise definitions of predictors and outcomes. 4) Moving from correlation to causality, the field needs more mechanistic studies and sufficiently powered longitudinal human studies. The latter could include frequent sampling studies to determine community dynamics, observational studies with clinically relevant endpoints, and intervention studies. These studies should ideally include quantification of total bacterial load and bacterial species (not relative abundance only), and measurement of all relevant microorganisms, biofilms, and host responses in the genital tract. 5) The efficacy of existing interventions, and pipeline of new interventions, are limited. We should expand the pipeline, which requires industry involvement and more investment into the field in general. We now have the tools to assess the effects of those interventions.

Disclosure No significant relationships.

\section{S12 - NEW APPROACHES FOR STI VACCINES}

\section{Tuesday, July 16, 2019 4:15 PM - 5:45 PM}

\section{S12.1 PROGRESS TOWARDS A GONORRHOEA VACCINE}

Kate L Seib*. Institute for Glycomics, Griffith University, Gold Coast, Australia

\subsection{6/sextrans-2019-sti.57}

Introduction Gonorrhoea is becoming increasingly difficult to control due to antibiotic resistance and the absence of a vaccine. However, a vaccine to the closely related bacteria Neisseria meningitidis, the outer membrane vesicle (OMV) vaccine MeNZB, was reported to be associated with reduced rates of gonorrhoea. To investigate this possible cross-protection we assessed serum raised to the meningococcal vaccine Bexsero, which contains the MeNZB OMV component plus three recombinant antigens (NadA, fHbp-GNA2091, NHBAGNA1030), in terms of its cross reactivity and functional activity against Neisseria gonorrhoeae.

Methods The similarity of MeNZB and Bexsero antigens to gonococcal proteins was assessed bioinformatically. Sera from rabbits immunised with the OMV component or the three recombinant antigens of Bexsero, and sera from humans immunised with Bexsero were tested in Western blot, ELISA, serum bactericidal activity (SBA) and neutrophil opsonophagocytic (OPA) assays to assess the level and functional activity of antibodies recognising $N$. gonorrhoeae.

Results There is a high level of sequence identity between MeNZB/Bexsero OMV antigens and gonococcal proteins. NHBA is the only Bexsero recombinant antigen that is conserved and surfaced exposed in N. gonorrhoeae. Rabbit antibodies to the OMV component or to the three recombinant antigens of Bexsero recognise gonococcal proteins and mediate SBA and OPA killing of N. gonorrhoeae. Furthermore, Bexsero induces antibodies in humans that recognise gonococcal proteins. Analysis of the functional activity of Bexsero-induced human antibodies against $N$. gonorrhoeae is ongoing.

Conclusions The ability of antibodies induced by MeNZB OMV proteins to recognise and kill $N$. gonorrhoeae could explain the decrease in gonococcal cases associated with MeNZB vaccination. The presence of antibodies to OMV proteins plus NHBA following Bexsero vaccination may result in additional protection against gonorrhoea. Analysis of Bexserovaccinate human sera may also facilitate the identification of a correlate of protection to guide future development of a gonococcal vaccine.

Disclosure No significant relationships.

\section{S12.2 A TRI-ANTIGEN PROTECTIVE SYPHILIS VACCINE DESIGN THAT ENHANCES TREPONEMAL CLEARANCE AND INHIBITS PATHOGEN DISSEMINATION}

Lorenzo Giacani*. University of Washington, Medicine - Allergy and Infectious Diseases, Seattle, USA

10.1136/sextrans-2019-sti.58

Background A protective vaccine against Treponema pallidum subsp. pallidum (T. pallidum) would facilitate syphilis control at a global scale. We have identified three T. pallidum antigens that, when used separately as immunogens, either attenuate chancre development at the challenge site or pathogen dissemination. Here, we report protection induced by a vaccine desing composed of these three antigens + two custom adjuvants containing Natural or Synthetic TLR4 agonists and a natural Mincle agonist.

Methods Recombinant TprK (aa 37-273), Tp0751 (aa 24237), and Subfamily I Tpr (aa 23-351) peptides were emulsified in either adjuvant and used to immunize groups of eight rabbits. Immunized animals were challenged intradermally with $10^{\wedge} 5 \mathrm{~T}$. pallidum/site at 10 sites, along with eight unimmunized control animals. Lesion development was recorded daily and treponemal burden measured by darkfield (DF) microscopy and qPCR of lesion aspirates and biopsies. Dissemination to distant tissues was evaluated by qPCR and rabbit infectivity test (RIT). 